\title{
The W7-X ECRH Plant: Recent Achievements
}

\author{
V. Erckmann ${ }^{1}$, P. Brand ${ }^{3}$, H. Braune ${ }^{1}$, G. Dammertz ${ }^{2}$, \\ G. Gantenbein ${ }^{2}$,W. Kasparek ${ }^{3}$, H. P. Laqua ${ }^{1}$, G. Michel ${ }^{1}$, M. Schmid ${ }^{2}$ \\ M. Thumm ${ }^{2}$, M. Weissgerber ${ }^{1}$, and the W7-X ECRH- teams at \\ IPP Greifswald ${ }^{1}$, FZK Karlsruhe ${ }^{2}$ and IPF Stuttgart ${ }^{3}$ \\ ${ }^{1}$ Max Planck Institut für Plasmaphysik, EURATOM Association, \\ Teilinstitut Greifswald, D-17491 Greifswald, Germany \\ 2 Forschungszentrum Karlsruhe, Association EURATOM-FZK, IHM, \\ Hermann-von-Helmholtz-Platz 1, D-76344 Eggenstein-Leopoldshafen, Germany, \\ ${ }^{3}$ Institut für Plasmaforschung, Universität Stuttgart, D-70569 Stuttgart, Germany
}

\begin{abstract}
The $10 \mathrm{MW}, 140 \mathrm{GHz}, \mathrm{CW}$ ECRH-plant for W 7-X is in an advanced state of commissioning and the installation was used to investigate advanced applications for extended heating- and current drive scenarios. The operation of the TED gyrotrons was recently extended to a $2^{\text {nd }}$ frequency of $103.6 \mathrm{GHz}$ at reduced output power and first results are presented. An improved collector sweep system for the W7-X gyrotrons with enhanced power capability and smooth power distribution was developed, results are reported.
\end{abstract}

Keywords: ECRH, Stellarator, Gyrotron, Microwaves

PACS: $52.50 . \mathrm{Sw}$ 84.40.Ik $52.55 . \mathrm{Hc}$

\section{INTRODUCTION}

W7-X (major radius $5.5 \mathrm{~m}$, minor radius $0.55 \mathrm{~m}$ ) is the next step in the Stellarator approach towards magnetic fusion power plants and aims at demonstrating quasi steady state operation at reactor relevant parameters. W7-X is equipped with a superconducting coil system, a continuously operating ECRH system and an actively pumped divertor for stationary particle and energy control. An ECRH-plant with 10 MW heating power at $140 \mathrm{GHz}$ in $\mathrm{CW}$ mode $(30 \mathrm{~min})$ is being built to support $\mathrm{W} 7-\mathrm{X}$ operation [1]. The standard heating and current drive scenario is X2- mode $\left(\mathrm{B}_{\text {res }}=2.5 \mathrm{~T}\right)$ with low field side launch (LFS). High-density operation above the X2 cut-off density of $1.210^{20} \mathrm{~m}^{-3}$ is accessible with O2-mode $\left(<2.510^{20} \mathrm{~m}^{-3}\right)$ and at even higher densities with O-X-B mode conversion heating [2]. X3-mode heating $\left(B_{\text {res }}=1.66 \mathrm{~T}, \mathrm{n}_{\mathrm{e}}<1.610^{20} \mathrm{~m}^{-3}\right)$ is a promising scenario for operation at reduced magnetic field [3]. A further extension of the operation range would be achieved with a two-frequency ECRH-system. We have therefore started to explore operation features of the W7-X gyrotrons beyond specifications, i.e. operation at a second frequency and improved power handling capability of the collector. 


\section{TWO FREQUENCY OPERATION}

The W7-X gyrotrons from both manufacturers TED and CPI were optimized for single frequency operation at $140 \mathrm{GHz}$. Full performance operation at $>0.9 \mathrm{MW}$ for $30 \mathrm{~min}$ was demonstrated with gyrotrons from both companies [4,5]. As the gyrotron diamond window has a resonant thickness of $4 \lambda / 2$ at $140 \mathrm{GHz}$, it is also transparent at $105 \mathrm{GHz}$ corresponding to $3 \lambda / 2$. Two resonant modes with the proper caustic radius, the TE 21,6 $(103.6 \mathrm{GHz})$ and $\mathrm{TE}_{22,6}(106.3 \mathrm{GHz})$ exist in the vicinity of the desired frequency. Calculations show, that both modes are converted with high quality into a Gaussian output beam by the quasi/optical mode converter in combination with the built-in 3mirror optics. Both modes could be exited by tuning the resonant magnetic field and adjusting the operation parameters $\left(\mathrm{I}_{\mathrm{beam}}=40 \mathrm{~A}\right.$ and $\left.\mathrm{U}_{\mathrm{acc}}=62 \mathrm{kV}\right)$. A slightly astigmatic output beam with a Gaussian mode content of about $97 \%$ was obtained in agreement with calculations.
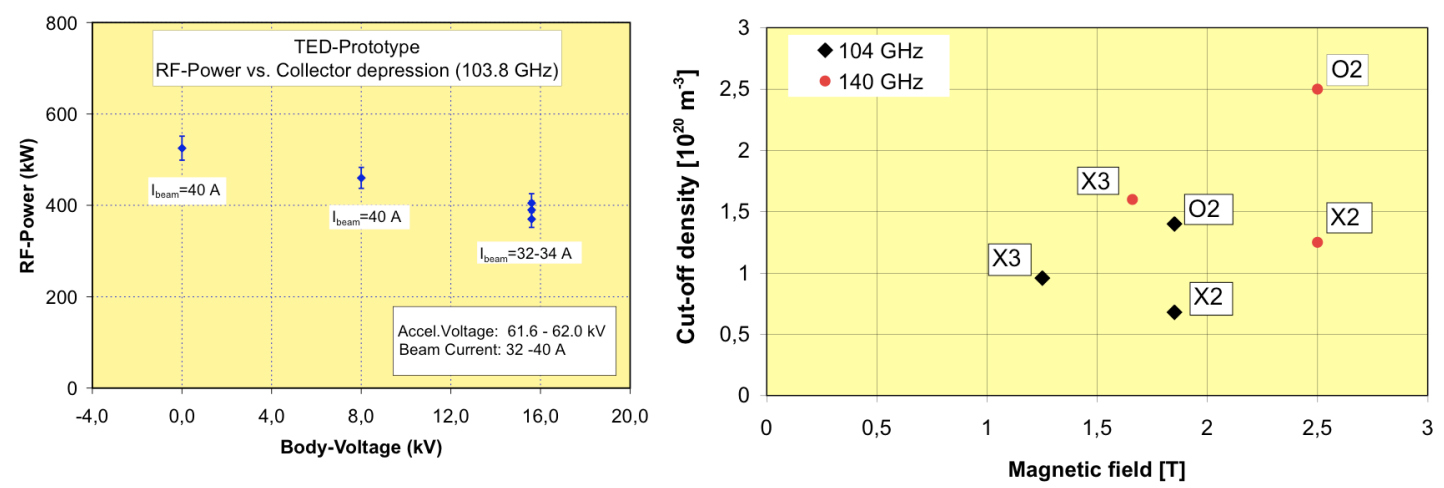

FIGURE 1. Left: Output power in the TE 21,6 mode $(103.6 \mathrm{GHz})$ as a function of the depression voltage at constant acceleration voltage $\left(\mathrm{U}_{\mathrm{ACC}}=62 \mathrm{KV}\right)$. Right: Cut-off density vs. resonant magnetic field for different modes at both frequencies.

We have focused on the TE 21,6 -mode operation, because the output beam was almost perfectly centered on the output window, whereas the beam from the TE 22,6 -mode was located somewhat off centre. As seen from Fig. 1, a maximum output power of about $0.52 \mathrm{MW}$ was achieved without collector voltage depression corresponding to an efficiency $\eta=21 \%$, which is slightly higher than the theoretical prediction of $\eta=17 \%$. The output power drops with increasing depression voltage while the efficiency increases from $21 \%$ to $27 \%$. The corresponding collector loading at 0 and $8 \mathrm{kV}$ depression voltage is 1.9 and $1.7 \mathrm{MW}$, respectively, which is incompatible with the collector-loading limit of $1.3 \mathrm{MW}$. Thus only operation with reduced beam current around $34 \mathrm{~A}$ (about $400 \mathrm{~kW}$ ) can be handled safely by the collector. The rf-beam was transmitted through 7 mirrors of the quasi-optical transmission line into a calorimetric cw-load. Transmission losses of about $20 \mathrm{~kW}$ were measured, which compares well with the transmission loss fraction at $0.9 \mathrm{MW}$, $140 \mathrm{GHz}$ operation. It is worth noting, that both the beam matching mirrors as well as the set of polarizers can be used without modification. Although the grooved polarizer mirror surfaces are matched to $140 \mathrm{GHz}$, the desired polarization can be adjusted by proper selection of the relative groove orientation. The larger beam size at the lower 
frequency is expected to introduce slightly higher losses as compared to the nominal frequency. On the other hand the atmospheric absorption is somewhat lower.

Assuming, that all series gyrotrons behave similar to the Prototype, ECRH for W7-X will be operated as a two-frequency system. As sketched in Fig. 2 (right), the operation range of experiments can then be extended towards different resonant magnetic field of 1.86 T (X2 and O2 mode) and 1.25 T (X3 mode), respectively. Once plasma start-up could be achieved with X3-mode, which is not clear yet, operation at $1.25 \mathrm{~T}$ is of particular interest, because ECRH could then provide a target plasma for NBI for high- $\beta$ physics studies, which is most promising at low magnetic field.

\section{OPTIMIZATION OF THE GYROTRON COLLECTOR LOADING}

State of the art MW-class CW-gyrotrons operate with an efficiency of typically $40-50 \%$. Thus 1-1.5 MW power remains in the electron-beam after the electron-wave interaction and must be dissipated in the collector. Vertical magnetic field sweeping systems (VFSS) became a standard technique, which keeps the specific heat dissipation at the collector within technically acceptable limits $\left(<5 \mathrm{MW} / \mathrm{m}^{2}\right)$ in the time average. As such collectors operate close to the technological limits, they represent a high-risk component with a small safety margin and often infer a power limit to gyrotron operation. To address this problem we have used the existing experimental set-up with an industrial TED gyrotron to investigate and optimize the power distribution of the electron beam along the collector surface. The beam spread (FWHM) without sweeping is about $50 \mathrm{~mm}$ in the vertical dimension leading to a power density at nominal operation of about $20 \mathrm{MW} / \mathrm{m}^{2}$, which is unacceptably high. All TED gyrotrons thus are equipped with a VFSS running at an optimized sweep frequency of $7 \mathrm{~Hz}$. A broad power deposition profile with strong peaking at the upper and lower turning points of the beam is achieved as shown in Fig. 2 (right).
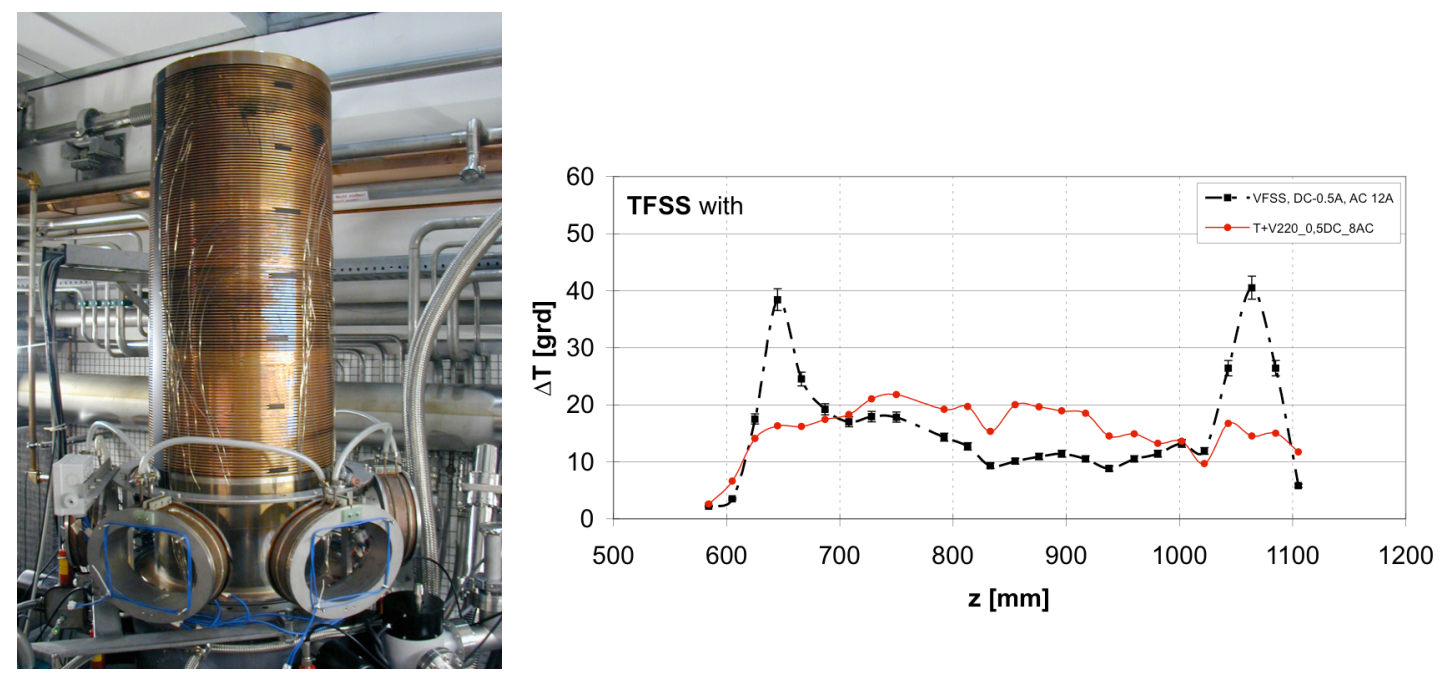

FIGURE 2: Left: The THALES gyrotron collector with the three pairs of TF-coils installed (VF-coil removed). Right: Profiles of the collector temperature increase $\Delta \mathrm{T}$ along the vertical coordinate $\mathrm{z}$ for combined collector sweeping (dots). The profile for VFSS only is also shown for reference (squares). 
The measurements are taken with an array of 49 thermocouples mounted at equal distances along the vertical direction of the collector. The temperature rise is measured as a function of vertical distance for the optimum sweep parameters. In principle, the power peaking could be avoided by running a saw-tooth like waveform. The ACmagnetic field, however, is strongly shielded by the copper cylinder of the collector, which affects particularly the higher Fourier harmonics and only the low frequency components can penetrate.

In a first step, we have performed experiments with a rotating transverse field sweeping system (TFSS), which was invented by IAP Nizhny Novgorod and applied for the first time at a Russian gyrotron [6]. The TFSS consists of 3 pairs of TF-coils, which are powered with a 3-phase AC-supply thus generating a transverse field, which rotates with $50 \mathrm{~Hz}$ [7]. The measurements were performed with reduced e-beam parameters (typically $0.75 \mathrm{MW}$, i.e. $15 \mathrm{~A}, 50 \mathrm{kV}$ ). The power peaking remained in the lower collector area and no advantage was gained with respect to the collector loading limits. In a next step, we have combined both, the TFSS and the VFSS. By proper tuning of both systems an almost perfectly flat power deposition profile along the collector area was obtained as seen in Fig. 2. A small DC-component was superimposed to control the position of the lower turning point. The peak loading is reduced by about a factor of 2 as compared to VFSS alone, which increases the safety margin of the present collector design significantly and/or may allow operation of the TED series tubes at higher electron beam currents (and possibly higher output power). The operation at $103.6 \mathrm{GHz}$ may become possible at higher power also. The results may also be important for the next generation gyrotrons with up to $2 \mathrm{MW}$ output power as being developed for ITER, because the existing collector design would already satisfy the e-beam heat dissipation demands.

\section{REFERENCES}

[1] V. Erckmann, H.J. Hartfuß, M. Kick, H. Renner, et al.: Proc. 17th IEEE/NPSS Symposium on Fusion Engineering, San Diego, USA (1997). Ed. IEEE, Piscataway, NJ 1998, 40 - 48

[2] H. P. Laqua, V. Erckmann, H.J. Hartfuß, H. Laqua, W7-AS Team, and ECRH Group: Phys. Rev. Lett. 78, 3467 (1997)

[3] V. Erckmann, P. Brand, H. Braune, G. Dammertz, et al., accepted for publ. in Fusion Science and Technology, 2007

[4] G. Dammertz, S. Alberti, A. Arnold, E. Borie, et al., IEEE Trans. Plasma Science 30 (2002) 808-818

[5] K. Felch, M. Blank, P. Borchard, P. Cahalan, S. Cauffman, T.S. Chu and H. Jory, Journal of Physics: Conference Series 25 (2005) pp. 13-23.

[6] privat communication

[7] G. Dammertz, S. Illy, B. Piosczyk, M. Schmid, D. Bariou, Proc. of the Joint 30th Int. Conf. on Infrared and Millimeter Waves and 13 th Int. Conf. On Terahertz Electronics, Williamsburg, USA, ISBN 0-7803-9349-X (2005) p 323-324 